\title{
Current and Emerging Clinical Applications of PSMA PET Diagnostic Imaging for Prostate Cancer
}

\author{
Andrea Farolfi, Letizia Calderoni, Francesco Mattana, Riccardo Mei, Sivi Telo, Stefano Fanti, and Paolo Castellucci \\ Nuclear Medicine Unit, IRCSS Azienda Ospedaliero-Universitaria di Bologna, Bologna, Italy
}

\begin{abstract}
Prostate-specific membrane antigen (PSMA) is highly expressed on most prostate cancer ( $\mathrm{PCa}$ ) cells, and several PSMA ligands for PET imaging are now available worldwide. ${ }^{68} \mathrm{Ga}-\mathrm{PSMA}-11$ has already received U.S. Food and Drug Administration approval, and use of PSMA PET is currently suggested by several international guidelines for investigating $\mathrm{PCa}$ in different clinical settings. In primary $\mathrm{PCa}$, PSMA PET has been shown to be superior to cross-sectional imaging for the detection of pelvic lymph nodes and distant metastases with subsequent clinical management changes. Additionally, it might also have a role in intraprostatic tumor localization, especially when combined with multiparametric MRI. In a setting of PCa recurrence, higher detection rates have been observed than for any other available imaging techniques, especially at low prostate-specific antigen values. Furthermore, PSMA PET consistently led to a shift in clinical management, thus increasing the proportion of radiotherapy, surgery, or other focal therapies at the expense of systemic options or no treatment. In oligometastatic disease after radical surgery, PSMA PET may be relevant in guiding a metastasis-directed therapy approach, as preliminary data seem to suggest a benefit in terms of progression-free survival after treatment of PSMA PET-positive lesions. As a staging and gatekeeping technique, PSMA PET represents a reliable wholebody imaging procedure in combination with second-line therapy of castration-resistant PCa, as well as being pivotal when assessing patients eligible for radioligand therapy such as ${ }^{177}$ Lu-PSMA. This critical review aims at providing a comprehensive overview of the latest literature on the current or emerging main indications, as well as a general outlook on the recommended interpretation criteria for PSMA PET imaging.
\end{abstract}

Key Words: genitourinary; molecular imaging; oncology; PSMA; positron emission imaging (PET); prostate cancer

J Nucl Med 2021; 62:596-604

DOI: $10.2967 /$ jnumed.120.257238

$\mathbf{P}$ rostate cancer $(\mathrm{PCa})$ is the most common malignancy in men and is associated with high morbidity and mortality rates (1). MRI and different PET radiotracers have been extensively used to improve the accuracy of conventional imaging, namely CT and bone scintigraphy, at all times during the natural history of PCa. Choline, labeled with either ${ }^{11} \mathrm{C}$ or ${ }^{18} \mathrm{~F}$, and ${ }^{18} \mathrm{~F}$-fluciclovine are still broadly used as metabolic PET tracers in clinical practice, and their role for imaging biochemical recurrence (BCR) and their impact on therapeutic management have been demonstrated in

Received Jan. 19, 2021; revision accepted Mar. 1, 2021

For correspondence or reprints, contact Andrea Farolfi (andrea.farolfi6@ unibo.it).

Published online Mar. 12, 2021

COPYRIGHT (C) 2021 by the Society of Nuclear Medicine and Molecular Imaging. clinical trials (2-5). Other PET tracers, such as gastrin-releasing polypeptide receptor-targeting radiopharmaceuticals, show promising results at various stages of $\mathrm{PCa}$, and data from prospective trials are awaited before translation of these tracers into clinical practice (6). The emerging data suggest that novel prostate-specific membrane antigen (PSMA)-based radioligands carry the highest diagnostic value in the imaging of $\mathrm{PCa}$ (7). PSMA is overexpressed in most PCa cells and is associated with higher prostatespecific antigen (PSA) values and a higher International Society of Urologic Pathologists (ISUP) grade at diagnosis, as well as with a worse overall survival. However, PSMA has shown a marked inter- and intrapatient heterogeneity $(8)$. The nuclear medicine community has come a long way since the first in-human applications of ${ }^{68}$ Ga-PSMA-11, which date back to 2012. Its approval by the U.S. Food and Drug Administration at production sites only in late 2020 marks an important step toward its wide acceptance, but this approval does not represent an endpoint to its further use in the molecular imaging of PCa (9). Prospective, randomized clinical trials incorporating PSMA imaging will probably soon be published; their results are needed to provide even more robust evidence of its role in improving patient outcome. Among PSMA ligands, some are based on small urea-based molecules and others are based on antibodies; some are bound with ${ }^{68} \mathrm{Ga}$ and others with ${ }^{18} \mathrm{~F}$ or other isotopes; and some PSMA ligands are for PET and others for SPECT (10-16). More radiolabeled PSMA ligands are expected in the future (Table 1). However, a detailed analysis of the differences in diagnostic performance for PSMA radiopharmaceuticals goes beyond the scope of this review. The aim of this critical review is to highlight use of the already established or currently emerging diagnostic applications of PSMA compounds during the natural history of PCa.

\section{INTRAPROSTATIC CANCER DETECTION}

The detection, characterization, and better definition of intraprostatic foci of $\mathrm{PCa}$ are among the most relevant emerging applications of PSMA PET imaging. In association with multiparametric MRI (mpMRI), PSMA PET may be used to detect the need for, and subsequently to guide, a targeted biopsy in patients presenting with clinically suspected PCa. Furthermore, PSMA PET might improve the accuracy of segmentation before radiation therapy or other localized treatments, offer a noninvasive characterization of unclear findings, and provide prognostic information.

\section{Biopsy Guidance}

mpMRI should be performed on all subjects presenting with clinically suspected PCa before any biopsy attempt in order to guide the biopsy to significant foci in accordance with the most recent recommendations (17). In this setting, PSMA PET might 
TABLE 1

PSMA Ligands Commonly Used in Clinical Practice for Imaging and Therapy

\begin{tabular}{|c|c|c|c|c|}
\hline Ligand & Label & Imaging or therapy & Advantages & Disadvantages \\
\hline $\begin{array}{l}\text { PSMA-11 (PSMA-HBED- } \\
\text { CC) (12) }\end{array}$ & ${ }^{68} \mathrm{Ga}$ & Imaging & $\begin{array}{l}\text { Most widely used in } \\
\text { literature; European } \\
\text { Medicines Agency } \\
\text { and Food and Drug } \\
\text { Administration } \\
\text { approval }\end{array}$ & $\begin{array}{l}{ }^{68} \text { Ga-related } \\
\text { disadvantages; high } \\
\text { accumulation in } \\
\text { urinary tract }\end{array}$ \\
\hline PSMA-617 (13) & ${ }^{68} \mathrm{Ga}$ & Imaging and therapy & $\begin{array}{l}\text { Reduced kidney uptake } \\
\text { compared with } \\
\text { PSMA-11 }\end{array}$ & $\begin{array}{l}{ }^{68} \text { Ga-related } \\
\text { disadvantages; } \\
\text { slightly slower tracer } \\
\text { kinetics than for } \\
\text { PSMA-11; high } \\
\text { accumulation in } \\
\text { urinary tract }\end{array}$ \\
\hline PSMA-I\&T (14) & ${ }^{68} \mathrm{Ga}$ & Imaging and therapy & $\begin{array}{l}\text { Reduced hepatic uptake } \\
\text { compared with } \\
\text { PSMA-11 }\end{array}$ & $\begin{array}{l}{ }^{68} \text { Ga-related } \\
\text { disadvantages; lower } \\
\text { lesion binding and } \\
\text { higher background } \\
\text { than for PSMA-11 }\end{array}$ \\
\hline DCFPyL (11) & ${ }^{18} \mathrm{~F}$ & Imaging & Low hepatic uptake & $\begin{array}{l}\text { High accumulation in } \\
\text { urinary tract }\end{array}$ \\
\hline PSMA-1007 (15) & ${ }^{18} \mathrm{~F}$ & Imaging & $\begin{array}{l}\text { Low accumulation in } \\
\text { urinary tract }\end{array}$ & $\begin{array}{l}\text { High hepatic uptake; } \\
\text { higher number of } \\
\text { PSMA-positive lesions } \\
\text { attributed to benign } \\
\text { origin* }^{*}\end{array}$ \\
\hline rhPSMA-7 (16) & ${ }^{18} \mathrm{~F},{ }^{68} \mathrm{Ga}$ & Imaging and therapy & $\begin{array}{l}\text { Radiohybrid concept; } \\
\text { low accumulation in } \\
\text { urinary tract with }{ }^{18} \mathrm{~F}\end{array}$ & $\begin{array}{l}\text { Higher number of } \\
\text { PSMA-positive lesions } \\
\text { attributed to benign } \\
\text { origin* }\end{array}$ \\
\hline
\end{tabular}

${ }^{*}$ Compared with ${ }^{68} \mathrm{Ga}-\mathrm{PSMA}-11$ (e.g., ganglia, unspecific bone lesions, unspecific lymph nodes).

increase the accuracy of mpMRI, mostly in patients with a high clinical suspicion when mpMRI results are negative (Prostate Imaging-Reporting and Data System 1-2) or inconclusive (Prostate Imaging-Reporting and Data System 3). Bodar et al. mapped foci of increased PSMA uptake within the prostate gland in 30 patients prospectively studied with ${ }^{18} \mathrm{~F}$-DCFPyL PET/CT before radical prostatectomy (RP) (18). The targeting of PSMA PET findings on a later biopsy showed PCa-positive lesions in 28 of 30 patients (93\%). However, considering all the intraprostatic cancer lesions, sensitivity and specificity for PSMA PET were $61.4 \%$ and $88.3 \%$, respectively. Chen et al. used PSMA PET and mpMRI alone or in a hybrid setting (PET/MRI) to improve the detection of clinically significant $\mathrm{PCa}$ in 54 men studied before RP, maintaining the final histopathology results as the standard of reference (19). Sixty-six lesions were retrospectively considered clinically significant. The combination of PET and MRI showed a significantly better accuracy than mpMRI alone: sensitivity was $89 \%$ versus $76 \%$, respectively $(P<0.01)$, and specificity was $96 \%$ versus $88 \%(P>$ $0.05)$. This improved accuracy was particularly evident when clinically significant lesions occurred within the context of a Prostate Imaging-Reporting and Data System score of 3.

From the limited literature data available, we can conclude that the use of PSMA PET could add diagnostic accuracy in patients with inconclusive MRI results. However, in consideration of the large number of patients who could benefit from PSMA PET and the still limited availability of this method, an extensive application of PSMA PET for this purpose does not appear to be easily feasible. Studies on highly selected populations could in the future clarify the role and the added value of PSMA PET in this context. The ongoing prospective multicenter PRIMARY clinical trial will measure and compare the sensitivity, specificity, positive predictive value, and negative predictive value of both mpMRI and PSMA PET versus targeted prostrate biopsy (20). The results will be used to determine the proportion of men who could safely avoid biopsy without compromising detection of clinically significant PCa.

\section{Segmentation for Radiation Therapy or Guidance of Other Focal Therapies}

Bettermann et al. showed a better accuracy for PSMA PET than for mpMRI for intraprostatic gross tumor volume (GTV) delineation. The authors prospectively performed PSMA PET and mpMRI on 17 patients who were candidates for RP (21). GTV contours for mpMRI and PSMA PET were drawn and compared with final GTVs drawn on histopathology images. Median tumor volumes were $10.4 \mathrm{~cm}^{3}$ for GTVs drawn on histology images, $10.8 \mathrm{~cm}^{3}$ for GTVs drawn on PSMA PET images, and $4.5 \mathrm{~cm}^{3}$ for mpMRI. Sensitivity and specificity were $86 \%$ and $87 \%$, respectively, for PSMA PET; $58 \%$ and $94 \%$, respectively, for mpMRI; and $91 \%$ and $84 \%$, respectively, for the combination of both techniques. 


\section{Characterization of Intraprostatic Findings and Prognostic Information}

Using a similar study design, Scheltema et al. retrospectively enrolled 56 patients who underwent mpMRI and PSMA PET before RP (22). PSMA PET was accurate in detecting prostate segments containing ISUP grade $2-3$, if compared with mpMRI, and it may have a role in diagnosing or monitoring PCa. Roberts et al. retrospectively enrolled 71 patients who had MRI-guided, biopsy-proven PCa and in whom PSMA PET was performed before surgery (23). PSMA uptake in the prostate has been correlated with adverse pathology outcomes and progression-free survival, with a minimum follow-up of 24 mo. PSMA PET provided reliable prognostic information, especially in patients with biopsy-proven Gleason $3+4$ disease potentially suitable for active surveillance or focal therapy.

On the basis of the available literature, and considering the anticipated widespread use of highly sensitive PET tomographs, we believe it is reasonable to suppose that PSMA PET will soon be a routine part of the diagnostic flowchart in many $\mathrm{PCa}$ patients before biopsy or primary therapy.

\section{STAGING}

A correct assessment of the tumor extension at onset is crucial to establish the correct therapeutic strategy after primary staging. In this setting, PSMA PET for lymph node and bone spread detection has shown high specificity and positive predictive values but a suboptimal sensitivity, which remains, however, significantly higher than that of conventional imaging (7). In the most recently published metaanalysis, the results of 11 studies, including 904 intermediate- or high-risk patients, were grouped: pooled sensitivity and specificity on a patient basis were $63 \%$ (95\% CI, 0.46-0.78) and 93\% (95\% CI, 0.88-0.96), respectively (24). On a lymph-node basis, they were $70 \%(95 \% \mathrm{CI}, 0.49-0.85)$ and $99 \%(95 \% \mathrm{CI}$, $0.96-1.00)$, respectively. The pooled positive predictive value and negative predictive value were above $80 \%$ whether in a per-patient or a per-node analysis. The recently published results of the proPSMA study are a game changer in staging high-risk PCa patients (25). The proPSMA study is the first multicentric, 2-arm, randomized study aimed at investigating whether PSMA PET may show an improved accuracy when compared with conventional imaging or if it may end up replacing conventional imaging as the only imaging method to perform in high-risk PCa patients at disease presentation. The study included 302 high-risk patients: 152 were randomly assigned to the conventional imaging diagnostic flowchart and 150 to PSMA PET only. The results were validated by a composite reference standard including histopathology, imaging, and laboratory data. At final diagnosis, 30\% of the patients showed local or distant metastatic disease. PSMA PET showed greater accuracy than conventional imaging, $92 \%$ versus $65 \%$, respectively; better sensitivity, $85 \%$ versus $38 \%$; higher impact on clinical decisions, $28 \%$ versus $15 \%$, and a lower number of indeterminate findings, $7 \%$ versus $23 \%$. The authors concluded that PSMA PET may replace conventional imaging when staging high-risk patients. Nevertheless, the main drawback is that in men undergoing radiotherapy, histologic confirmation of nodal disease was not performed, and some patients might have had microscopic disease that was missed by either conventional imaging or PSMA PET. Wondergem et al. studied 160 high-risk patients at presentation with a fluorinated PSMA compound ( ${ }^{18} \mathrm{~F}$-DCFPyL) (26). PSMA PET correctly identified 81 of $90(90 \%)$ patients with local or distant metastatic spread at final diagnosis. PSMA PET detected additional lymph node metastases in almost all patients (41/42) for whom CT was already positive in at least 1 lymph node. PSMA PET determined a significant shift in patient management in $17 \%$ of the population. In accordance with the proPSMA study, the authors concluded that PSMA PET might be considered the first-line imaging modality for high-risk PCa at presentation, with no need for further diagnostics (Supplemental Fig. 1; supplemental materials are available at $\mathrm{http}: / /$ jnm.snmjournals.org). In a recent prospective multicenter single-arm, open-label phase 3 trial, the accuracy of PSMA PET in the detection of N1 status was assessed in 277 intermediate- or high-risk patients at presentation (27). At final diagnosis, $27 \%$ of the patients were $\mathrm{N} 1$ at histopathology. On a region-based analysis, sensitivity and specificity by PSMA PET in N1 detection were $40 \%$ and $95 \%$, respectively. Higher PSA values and larger nodes were correlated with increased sensitivity by PSMA PET. According to the available data and the foreseeable increase in PSMA PET use before primary treatment, it stands to reason that future inclusion of PSMA PET within the main international guidelines can be expected, at least in a setting of high-risk PCa at disease presentation. Moreover, a cost-effectiveness analysis developed using data from the proPSMA study demonstrated greater accuracy and lower direct comparative costs for PSMA PET than for conventional imaging, namely CT and bone scanning (28).

\section{BIOCHEMICAL RECURRENCE}

Imaging in PSA persistence or recurrence after radical treatment aims at treatment changes and thus possibly a better clinical outcome. PSMA PET demonstrated higher sensitivity than ${ }^{11} \mathrm{C}$-choline or ${ }^{18}$ F-fluciclovine PET in this setting $(29,30)$, and scan positivity increases with higher PSA values (7). A common limitation of PSMA PET for this purpose is the lack of robust validation of PSMA PET-positive findings and lack of accurate evaluation of its impact on outcome, since most of the data are retrospective or with a short median follow-up time. However, numerous data confirm a significant impact of PSMA PET at least on clinical management. A metaanalysis investigating the impact of PSMA PET on management of BCR patients (11 studies, 908 patients) reported changes in $54 \%$ of patients, although substantial heterogeneity among the included studies was noted - that is, differences in clinical settings, types of initial definitive treatment, and baseline characteristics (31). Between $5 \%$ and $20 \%$ of men continue to have detectable PSA after RP (most often defined as PSA $\geq 0.1-0.2 \mathrm{ng} / \mathrm{mL}$ within $4-8 \mathrm{wk}$ from surgery). This condition is often associated with poor prognosis. In this patient setting, retrospective studies report a PSMA PET positivity rate ranging from $67 \%$ to $70 \%(32-34)$. According to the European Association of Urology guidelines, PSMA PET is the most sensitive imaging modality to detect metastasis in this patient setting and should be offered to patients with a PSA higher than $0.2 \mathrm{ng} / \mathrm{mL}$ after RP (Table 2) (35). In a large single-arm, multicenter prospective study, 635 patients with BCR after RP (41\%), radiation therapy $(27 \%)$, or both $(32 \%)$ were enrolled, with the main aim of evaluating the positive predictive value and the detection rate of PSMA PET (36). PSMA PET showed recurrent PCa in $75 \%$ of patients. The positive predictive value was 0.84 in the 87 patients validated by histopathology and 0.92 in the 217 patients validated by the composite reference standard. As expected, the PSMA PET detection rate was associated with increased PSA values, ranging from $38 \%$ in patients with a PSA lower than $0.5 \mathrm{ng} / \mathrm{mL}$ to $97 \%$ in those with a PSA higher than $5.0 \mathrm{ng} / \mathrm{mL}$. These data confirm that higher serum PSA levels are associated with PSMA positivity in BCR. Careful 
TABLE 2

2020 Recommendations on Use of PSMA PET or Next-Generation Imaging (NGI; i.e., PET/CT, PET/MRI and Whole-Body MRI) ${ }^{\star}$

\begin{tabular}{|c|c|c|c|c|}
\hline Clinical stage & $\begin{array}{l}\text { EAU-EANM-ESTRO-ESUR- } \\
\text { SIOG }(17,35)\end{array}$ & $\begin{array}{l}\text { Strength } \\
\text { rating }\end{array}$ & $\operatorname{ASCO}(40)$ & $\begin{array}{l}\text { Strength } \\
\text { rating }\end{array}$ \\
\hline Diagnosis & Not recommended & & Not recommended & \\
\hline Staging & $\begin{array}{l}\text { PSMA PET; not } \\
\text { recommended; perform at } \\
\text { least cross-sectional } \\
\text { imaging for intermediate- } \\
\text { and high-risk patients }\end{array}$ & & $\begin{array}{l}\text { When conventional imaging is negative } \\
\text { in patients with high risk of } \\
\text { metastatic disease, NGI may add } \\
\text { clinical benefit, although prospective } \\
\text { data are limited; when conventional } \\
\text { imaging is suggestive or equivocal, } \\
\text { NGl may be offered to patients for } \\
\text { clarification of equivocal findings or } \\
\text { detection of additional sites of } \\
\text { disease, which could potentially alter } \\
\text { management, although prospective } \\
\text { data are limited }\end{array}$ & Weak \\
\hline $\mathrm{BCR}$ & $\begin{array}{l}\text { Perform PSMA PET/CT if PSA } \\
>0.2 \mathrm{ng} / \mathrm{mL} \text { and if results } \\
\text { will influence subsequent } \\
\text { treatment decisions }\end{array}$ & Weak & $\begin{array}{l}\text { Goal of therapy and potential use of } \\
\text { salvage local therapies in these } \\
\text { scenarios should guide choice of } \\
\text { imaging }\end{array}$ & Moderate \\
\hline PSA persistence & $\begin{array}{l}\text { Offer PSMA PET to men with } \\
\text { persistent PSA }>0.2 \mathrm{ng} / \mathrm{mL} \\
\text { to exclude metastatic } \\
\text { disease }\end{array}$ & Weak & $\begin{array}{l}\text { For men for whom salvage local or } \\
\text { regional therapy is contemplated, } \\
\text { there is evidence supporting NGl for } \\
\text { detection of local or distant sites of } \\
\text { disease }\end{array}$ & Moderate \\
\hline $\begin{array}{l}\text { Before salvage } \\
\text { radiation } \\
\text { therapy }\end{array}$ & $\begin{array}{l}\text { Perform PSMA PET/CT (if } \\
\text { available) or }{ }^{18} \mathrm{~F} \text {-fluciclovine } \\
\text { or choline in patients fit for } \\
\text { curative salvage treatment }\end{array}$ & Strong & $\begin{array}{l}\text { For men for whom SRT is } \\
\text { contemplated, PSMA imaging should } \\
\text { be offered (or NGI) as it has superior } \\
\text { disease detection performance } \\
\text { characteristics and may alter patient } \\
\text { management }\end{array}$ & High \\
\hline $\begin{array}{l}\text { Nonmetastatic } \\
\text { CRPC }\end{array}$ & $\begin{array}{l}\text { With more sensitive imaging } \\
\text { techniques such as PSMA } \\
\text { PET/CT or whole-body MRI, } \\
\text { more patients are expected } \\
\text { to be diagnosed with early } \\
\text { metastatic CRPC }\end{array}$ & Not reported & $\begin{array}{l}\text { For men with nonmetastatic CRPC, } \\
\mathrm{NGl} \text { can be offered only if change in } \\
\text { clinical care is contemplated }\end{array}$ & Moderate \\
\hline Metastatic CRPC & $\begin{array}{l}\text { Use of choline or PSMA PET/ } \\
\text { CT scans for progressing } \\
\text { CRPC is unclear and most } \\
\text { likely not as beneficial as for } \\
\text { patients with BCR or } \\
\text { hormone-naïve disease }\end{array}$ & Not reported & $\begin{array}{l}\text { Use of NGI in this cohort is unclear, } \\
\text { with paucity of prospective data. } \\
\text { When a change in clinical care is } \\
\text { contemplated and there is a high } \\
\text { clinical suspicion of subclinical } \\
\text { metastasis despite negative } \\
\text { conventional imaging, the use of NGI } \\
\text { could be contemplated. In clear } \\
\text { evidence of radiographic progression } \\
\text { on } \mathrm{Cl} \text {, NGI should not be routinely } \\
\text { offered. NGI may play role if } \\
\text { performed at baseline to facilitate } \\
\text { comparison of imaging findings/ } \\
\text { extent of progression of disease }\end{array}$ & Insufficient \\
\hline
\end{tabular}

*According to the guidelines of the European Association of Urology (EAU), European Association of Nuclear Medicine (EANM), European Society for Radiotherapy and Oncology (ESTRO), European Society of Urogenital Radiology (ESUR), and International Society of Geriatric Oncology (SIOG) and the guidelines of the American Society of Clinical Oncology (ASCO).

patient selection using nomograms has been proposed to maximize the probability of a positive PSMA PET result, implementing clinical parameters such as ISUP grades, current androgen-deprivation therapy (ADT), time to BCR, clinical stage, and PSA kinetics, with areas under the receiver-operating-characteristic curve ranging from 0.69 to $0.76(37,38)$. In one study, Rauscher et al. included 272 hormone-sensitive patients with previous RP and PSA values between 0.2 and $1.0 \mathrm{ng} / \mathrm{mL}$ at the time of PSMA PET (37). Among those, about $10 \%$ were on ADT at the time of the PSMA PET scan. In a multivariable regression model, ADT administration and PSA values were identified as the most relevant predictors of positive PSMA PET results. Similarly, Ceci et al. included 703 patients with PSA failure after RP, stratified according to different clinical settings, that is, first BCR, recurrence after salvage treatment, PSA persistence after radical surgery, and advanced stage of PCa before second-line systemic therapy (38). In a multivariable regression model, ISUP 
grade, PSA values, PSA doubling time, and clinical setting were independent predictors of a positive PSMA PET result.

We can conclude that besides PSA values at the time of PSMA PET, concurrent ADT and PSA kinetics were the most relevant predictors of a positive scan in BCR patients. Nevertheless, in clinical daily practice, despite the high detection rates and accurate patient selection, a not-negligible number of patients will have a negative PSMA PET result. A prospective multicenter study (39) was performed to evaluate the predictive value of PSMA PET in 260 men with BCR (PSA of $0.26 \mathrm{ng} / \mathrm{mL}$; follow-up of $38 \mathrm{mo}$ ) who were candidates for salvage radiotherapy (SRT). Overall, freedom from progression after $3 \mathrm{y}$ was statistically significantly increased in patients with negative PSMA PET results or when PSMA PET showed disease confined to the prostatic fossa, in comparison with patients showing extraprostatic disease $(P<0.0001)$. It is interesting that in the same population, PSA values were not able to stratify patients with the same statistically significant accuracy.

\section{SRT PLANNING}

SRT after RP is associated with PSA control in about $50 \%$ of patients. International guidelines suggest performing SRT when serum PSA levels are lower than $0.5 / 1.0 \mathrm{ng} / \mathrm{mL}$ (35). At these PSA levels, conventional imaging demonstrated very low sensitivity in detecting sites of recurrence. For this reason, GTVs are usually drawn without imaging guidance. PSMA PET in patients eligible for SRT may improve the likelihood of PSA response and is suggested by the main international guidelines (Table 2) (35,40). Calais et al. enrolled 270

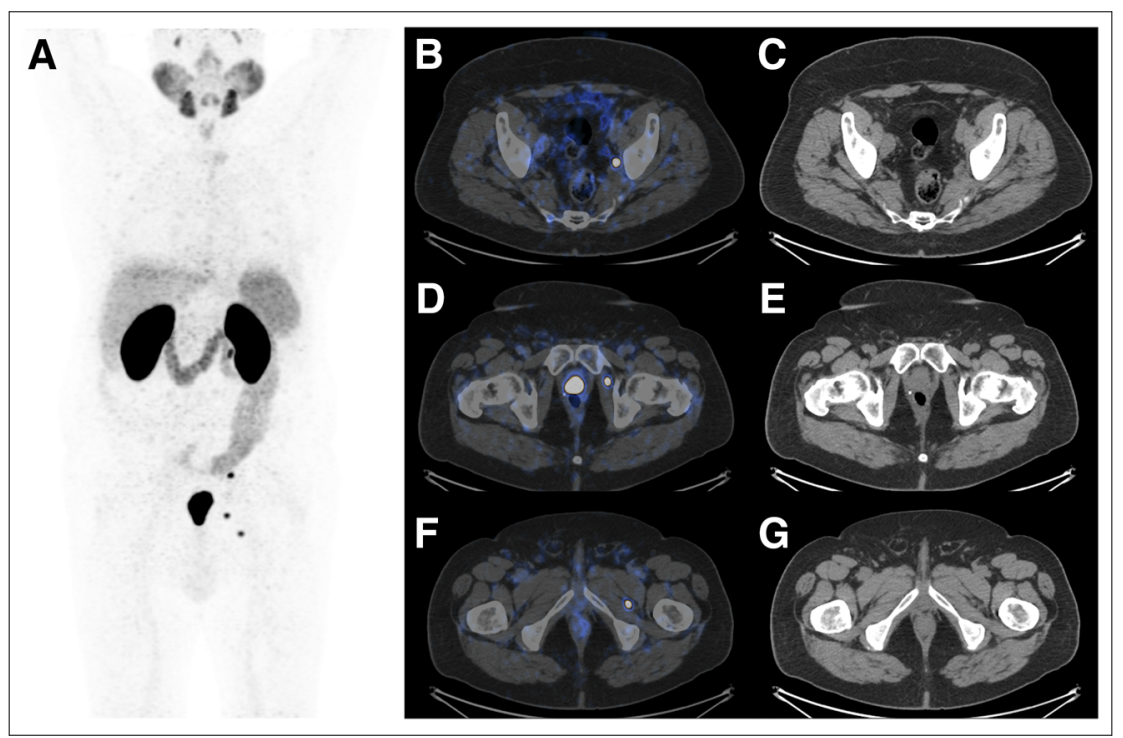

FIGURE 1. PSMA-guided salvage treatment in $69-y$-old man with initial PSA level of $5.1 \mathrm{ng} / \mathrm{mL}$, who underwent robot-assisted laparoscopic prostatectomy and lymph node dissection for adenocarcinoma with neuroendocrine phenotype, ISUP 4, pT3a pNO (0/11), R0. PSA persistence existed 4 wk after surgery, at $0.73 \mathrm{ng} / \mathrm{mL}$. Patient was referred for PSMA PET before scheduled SRT. PSA level at time of scan was $0.92 \mathrm{ng} / \mathrm{mL}$, with PSA doubling time of $5.2 \mathrm{mo}$. (A) PSMA PET maximumintensity projection. (B-G) PSMA PET/CT images and CT images showing 1 right obturator lymph node with PSMA uptake (B and C) and 2 intramuscular lymph nodes with PSMA uptake (D-G). Patient was treated with SRT and simultaneous integrated boosts and showed complete PSA response 9 mo after treatment. B, D, and F are PET/CT images, and C, E, and G are CT images. Image in $B(P E T / C T)$ shows the presence of a left obturator lymph node (PET-positive); in C (same slice, CT only), the same lymph node is not visible (short-axis diameter $<10 \mathrm{~mm}$ ). Image in D (PET/ CT) shows the presence of a left intramuscular lymph node (PET-positive); in E (same slice, CT only), the same lymph node is not visible (short-axis diameter $<10 \mathrm{~mm}$ ). Image in F (PET/CT) shows the presence of a left intramuscular lymph node (PET-positive); in G (same slice, CT only), the same lymph node is not visible (short-axis diameter $<10 \mathrm{~mm}$ ). patients after prostatectomy and before SRT who underwent PSMA PET at a PSA level of less than $1 \mathrm{ng} / \mathrm{mL}$ (median, $0.48 \mathrm{ng} / \mathrm{mL}$ ) pers $19 \%$ of the patients (Fig. 1). In a randomized phase 3 trial aimed to evaluate the success rate of SRT with and without radiation therapy planning based on PSMA PET findings, the primary endpoint was the SRT success rate, measured as biochemical progression-free surrollment is standard SRT, and 102 patients in the investigational arm underwent PSMA PET before SRT planning. Patients in the control group were staged heterogeneously using ${ }^{18} \mathrm{~F}$-fluciclovine PET (33\%), CT an no imaging. In the intervention group, PSMA PET This large prospective study will provide useful information for SRT and whether the impact of PSMA PET on SRT planning would translate into better patient outcomes.

\section{METASTASIS-DIRECTED THERAPIES AFTER RADICAL TREATMENT}

The oligometastatic state is proposed as a stage of cancer spread intermediate between localized and systemic disease, enabling a potential opportunity for metastasis-directed therapy to delay the emergence of polymetastatic disease (44). Disease volume and distribution have prognostic implications for patient management, quality of life, and survival, and thus, prompt recognition of oligometastatic $\mathrm{PCa}$ is desirable (45). However, the type of imaging that best defines oligometastatic PCa for the purpose of metastasis-directed therapy is debated (46). PSMA-based literature on this topic is mostly retrospective, and the randomized phase 2 ORIOLE (Observation vs. Stereotactic Ablative Radiation for Oligometastatic Prostate Cancer) study is the first clinical trial on PSMA PET-directed salvage therapy (47). Thirty-six patients with hormone-sensitive oligometastatic PCa underwent conventional imaging and were randomized to receive SBRT versus observation alone. Baseline PSMA PET was performed with ${ }^{18} \mathrm{~F}$ DCFPyL, and PSMA PET results were not used for SBRT treatment planning. At baseline PSMA PET, 16 of 36 (44.4\%) patients treated with SBRT showed positive findings that were not included in the prescribed treatment fields. Post hoc analysis of progression-free survival based on extent of untreated disease appreciable by PSMA PET found improved progression-free survival and distant metastasis-free survival advantages among men who received consolidation therapy for all PSMA-avid disease 
(hazard ratio, 0.26; 95\% CI, 0.09-0.76; $P=0.006$ ). This means that PSMA PET should be considered for metastasis-directed therapy to maximize patient benefit in oligometastatic PCa and that consolidation therapy in cases of PSMA PET positivity might improve progression-free survival.

\section{CASTRATION-RESISTANT PCA (CRPC)}

In CRPC, the number of available treatments is steadily rising over ADT, ranging from novel androgen receptor-targeted therapy (abiraterone, enzalutamide, or apalutamide) to anti-programmed cell death protein 1 and radionuclide therapy. In this setting, conventional imaging is recommended (35) despite the emergence of PSMA PET as an accurate imaging modality for evaluating CRPC patients. A multicenter retrospective study including 200 patients with a PSA level higher than $2.0 \mathrm{ng} / \mathrm{mL}$, negative results on conventional imaging, and a high risk for metastasis (i.e., PSA doubling time $\leqq 10$ mo or Gleason score $\geq 8$ ) aimed to assess the performance of PSMA PET in nonmetastatic CRPC (48). PSMA PET was positive in 196 of 200 (98\%) patients. Overall, PSMA PET showed pelvic disease in $44 \%$, including $24 \%$ with local prostate bed recurrence, and distant metastasis in $55 \%$ despite negative findings on conventional imaging (Supplemental Fig. 2). The overall accuracy of PSMA PET was $95 \%$ for osseous lesions and $60 \%$ for soft-tissue lesions. PSMA PET also demonstrated a shift in $30 \%$ of patients regarding per-patient Prostate Cancer Clinical Trials Working Group clinical subtype in comparison with conventional imaging, as well as a major concordance with conventional imaging, in a multicenter retrospective analysis of 67 CRPC patients imaged with PSMA PET and CT plus bone scintigraphy or whole-body MRI (49). According to these results, it stands to reason that PSMA PET leads to an earlier detection of metastasis than does conventional imaging and a change in clinical subtype, which may trigger earlier or different treatments. However, whether there could be an impact on overall survival or quality of life has yet to be determined, and further studies are warranted. Additionally, PSMA PET might be useful for selecting patients for the most appropriate treatment. In a retrospective analysis of 80 advanced-CRPC patients treated with ${ }^{223} \mathrm{Ra}$-dichloride, the final outcome was significantly better in the group of patients studied with PSMA PET before treatment than in those who were staged only with conventional imaging (50). Moreover, assessing PSMA expression is essential for the inclusion criteria in all PSMAbased radioligand therapy trials, since some patients may show low or absent PSMA expression, which is a contraindication for radioligand therapy. Experience derived from ${ }^{177}$ Lu-PSMA suggests a dual-tracer approach using both PSMA and ${ }^{18} \mathrm{~F}-\mathrm{FDG}$ for patient selection before treatment $(51,52) .{ }^{18} \mathrm{~F}-\mathrm{FDG}$-avid disease represents sites of aggressive disease that cannot efficiently be targeted with radioligand therapy. However, an optimal threshold for defining low PSMA expression on PSMA PET has not been defined or validated yet. Further prospective trials are required to elucidate the role of PSMA PET in response assessment and survival prediction. In a retrospective study on PSMA PET before and after 3 cycles of docetaxel, 16 metastatic CRPC patients were evaluated (53). The authors compared PSA decline with the responses on PSMA PET and CT. PSMA PET was better than $\mathrm{CT}$ as a predictor of response (56\% of the cases for PSMA PET vs. $33 \%$ for CT). In another retrospective study, 43 patients with metastatic CRPC underwent PSMA PET before and after systemic therapies (54). PSMA PET parameters, as well as RECIST 1.1 (55), were significantly associated with PSA response. However, neither the investigated PET parameters nor PSA level or RECIST 1.1 were associated with overall survival. This result could be explained by the design of the study and the heterogeneity of treatments and by the lack of standardized criteria to assess response (or progression) at PSMA PET. In this regard, the PSMA PET Progression Criteria were proposed to define disease progression (56), since the criteria of the Prostate Cancer Clinical Trials Working Group include only laboratory parameters and conventional imaging but no molecular imaging (57). The proposed definition of PSMA PET progression in metastatic CRPC is reported in Supplemental Table 1.

\section{ANTIANDROGEN MODULATION OF PSMA}

PSMA PET is routinely performed on many patients who have received or are receiving $\mathrm{ADT}$ at the time of the investigation; thus, the potential interaction of ADT on PSMA expression should be fully investigated, with implications for image interpretation and PSMA radioligand therapy timing. In vitro studies evaluating the effect of ADT on PSMA expression were first published by Wright et al. (58). Two elements regulate PSMA expression: PSMA promoter and PSMA enhancer, located within the third intron of FOLH1. FOLH1 gene expression is downregulated by androgens that reduce transcription of PSMA messenger RNA. On the other hand, antiandrogen administration upregulates the FOLH1 gene, causing an increased PSMA expression. In vivo studies showed that the PSMA expression is increased after ADT whereas the tumor size is decreased after administration of enzalutamide (59). Therefore, theoretically, the effect of ADT on images may be an increased PSMA expression before reduction in tumor size. Summarizing, we can say that ADT administration may lead to an increased PSMA uptake due to androgen receptor inhibition but that androgen receptor inhibitors may also lead to a reduction in tumor mass with consequent PCa cell death. Hope et al. showed that PSMA uptake significantly increased in 1 hormonal therapy-naïve patient imaged with PSMA PET before and after 4 wk of ADT (single administration of $7.5 \mathrm{mg}$ of leuprolide and 50 $\mathrm{mg}$ of bicalutamide/every day), in contrast to a significant reduction in PSA level, which dropped from 66 to $9 \mathrm{ng} / \mathrm{mL}$ (60). Moreover, some authors have postulated that modulation of PSMA expression after ADT differs depending on the status of the patient: CRPC or castration-sensitive PCa (CSPC). In this regard, Emmett et al. studied with sequential PSMA PET 8 CSPC patients at baseline and after 9, 18, and $28 \mathrm{~d}$ from ADT administration (luteinizing hormone-releasing hormone plus bicalutamide) (61). They also enrolled 7 CRPC patients studied with PSMA PET at the same time points after administration of abiraterone or enzalutamide. After $9 \mathrm{~d}$, luteinizing hormone-releasing hormone plus bicalutamide stimulation caused a median 30\% reduction in PSMA uptake in CSPC patients, whereas in CRPC patients, abiraterone/ enzalutamide administration caused a median $45 \%$ increased PSMA expression. According to these data, it could be postulated that PSMA expression after ADT stimulation differs depending on the patient status. The authors concluded that there is a rapid dichotomous response to ADT depending on the presence of a CSPC or CRPC phenotype. If this hypothesis is correct, PSMA PET could be used in the future for early classification of patients after a few days of hormonal treatment. In a prospective clinical trial aimed to understand if ADT administration may improve the 
performance of PSMA PET in PCa patients at presentation, 9 treatment-naïve patients were enrolled (62). PSMA PET/MRI has been performed at baseline and 3 times after administration of ADT in a course ranging from 1 to $8 \mathrm{wk}$. The authors observed a heterogeneous increase in PSMA uptake after 3-4 wk of ADT administration, whereas of those lesions with decreasing PSMA uptake in terms of $\mathrm{SUV}_{\max }$, none disappeared. It is interesting that this finding was more evident in bone metastases. According to these data, the optimal imaging time point to perform a second PSMA PET exam might be 3-4 wk after ADT administration. Finally, Afshar-Oromieh et al. studied the effect of long-term ADT (mean, $7 \mathrm{mo}$ ) in $10 \mathrm{CSPC}$ patients (63). PSMA uptake decreased in about $75 \%$ of the lesions, whereas in a small proportion of lesions (13\%) PSMA uptake increased despite a complete or partial PSA response. The authors postulated that the lesions that showed an increased uptake despite a clinical and PSA response might correlate with those cell clones that become castration-resistant first. Summing up briefly, considering the paucity of literature about this topic, we can probably assume that in CSPC patients, a short duration of ADT administration may increase PSMA expression whereas long-term ADT might have the opposite effect, even if it is possibly able to allow early detection of those lesions at risk of becoming castration-resistant. We can conclude by saying that there are still many unanswered questions and that foremost among these is the question of the optimal time point between ADT administration and PSMA imaging to reduce or increase (depending on the clinical needs) the effect of ADT on PSMA expression (64). In addition, further prospective studies are needed to clarify the influence of ADT administration on PSMA expression and the impact on PET imaging.

\section{STANDARDIZED REPORTING AND INTERPRETATION}

With the increasing diffusion of PSMA PET imaging worldwide, the application of standardized, unique methods to read and interpret images has become mandatory in order to collect reproducible data and increase the accuracy of PSMA PET. Several criteria have been already proposed, and Table 3 summarizes the key features.

\section{PROMISE Criteria}

The PROMISE criteria are a suggested standardization for PSMA PET both for reading based on the intensity of PSMA expression (known as miPSMA scores) and for interpretating the images and staging the disease (known as miTNM scores) (65). miPSMA categories were defined in relation to mean PSMA uptake in the blood pool, the liver, and the parotid gland, ranging from 0 to 3 . However, the authors of the criteria recommend that image interpretation and the conclusion on disease extent (miTNM scoring) be performed within the clinical context and with consideration of the extent and the location of PET findings. miTNM scores could be used as a guide for a standardized report taking into consideration the presence, location, and extent of local PCa and the pattern of metastases; the PSMA expression level of tumor lesions; and the diagnostic confidence about the reported findings.

TABLE 3

Proposed Reading and Interpretation Criteria by PSMA RADS (66), E-PSMA (67), and PROMISE (65) for PSMA PET

\begin{tabular}{|c|c|c|c|}
\hline Parameter & PSMA RADS (66) & E-PSMA (67) & PROMISE (65) \\
\hline $\begin{array}{l}\text { PSMA PET } \\
\text { reading score }\end{array}$ & & $\begin{array}{l}\text { E-PSMA scores*: 0-3; 0: } \\
\text { uptake < blood pool; } 1 \text { : } \\
\text { uptake }>\text { blood pool and }< \\
\text { liver; } 2 \text { : uptake }>\text { liver and }< \\
\text { parotid glands; } 3 \text { : uptake }> \\
\text { parotid glands }\end{array}$ & $\begin{array}{l}\text { miPSMA scores }^{\dagger}: 0-3 ; 0: \\
\text { uptake }<\text { blood pool; } 1 \text { : } \\
\text { uptake }>\text { blood pool and }< \\
\text { liver; } 2 \text { : uptake }>\text { liver and }< \\
\text { parotid glands; } 3 \text { : uptake }> \\
\text { parotid glands }\end{array}$ \\
\hline $\begin{array}{l}\text { PSMA PET } \\
\text { interpretation }\end{array}$ & $\begin{array}{l}\text { 1A: benign lesion without } \\
\text { abnormal uptake; 1B: benign } \\
\text { lesion with abnormal uptake; } \\
\text { 2: likely benign; 3: equivocal; } \\
\text { 3A: equivocal uptake in soft- } \\
\text { tissue site typical of PCa } \\
\text { involvement; 3B: equivocal } \\
\text { uptake in bone lesion not } \\
\text { definitive but also not } \\
\text { atypical of PCa on anatomic } \\
\text { imaging; 3C: intense uptake } \\
\text { in site highly atypical of all } \\
\text { but advanced stages of } \\
\text { PCa; 3D: lesion suggestive } \\
\text { of malignancy on anatomic } \\
\text { imaging but lacking uptake; } \\
\text { 4: PCa highly likely, with } \\
\text { intense uptake in site typical } \\
\text { of PCa but lacking definitive } \\
\text { findings on conventional } \\
\text { imaging; } 5 \text { : PCa almost } \\
\text { certainly present }\end{array}$ & $\begin{array}{l}\text { 1: benign lesion without } \\
\text { abnormal PSMA uptake; } 2 \text { : } \\
\text { probably benign lesion, with } \\
\text { faint PSMA uptake in site } \\
\text { atypical of PCa; } 3 \text { : equivocal } \\
\text { finding, with faint uptake in } \\
\text { site typical of PCa or intense } \\
\text { uptake in site atypical of } \\
\text { PCa; } 4 \text { : probably PCa, with } \\
\text { intense uptake in site typical } \\
\text { of PCa but without definitive } \\
\text { findings on CT; } 5 \text { : definitive } \\
\text { evidence of PCa, with } \\
\text { intense uptake in site typical } \\
\text { of PCa and definitive } \\
\text { findings on CT }\end{array}$ & $\begin{array}{l}\text { Scores } 2 \text { and } 3 \text { should be } \\
\text { considered PCa lesions, } \\
\text { depending on clinical } \\
\text { context, extent, and location } \\
\text { of findings }\end{array}$ \\
\hline
\end{tabular}

${ }^{*}$ Designed for most extensively used PSMA ligands $\left({ }^{68} \mathrm{Ga}-\mathrm{PSMA}-11,{ }^{18} \mathrm{~F}-\mathrm{DCFPyL}\right.$, and $\left.{ }^{18} \mathrm{~F}-\mathrm{PSMA}-1007\right)$.

†Designed mainly for ${ }^{68} \mathrm{Ga}$-PSMA-11. For PSMA ligands with liver-dominant excretion, spleen is recommended as reference organ instead of liver. 


\section{PSMA RADS}

PSMA RADS is a classification of PSMA PET findings into categories that reflect the likelihood of the presence of PCa (66). Like the other radiologic RADS criteria, the goal of PSMA RADS is to score the level of confidence of the reader on the presence of $\mathrm{PCa}$ and the potential need for any additional work-up. The scores for PSMA RADS range from 1 to 5, with higher numbers indicating a greater probability of PCa. In addition, the authors of the classification also recommend that a complete clinical history be collected for each patient, including the current and previous PSA levels, the findings of other imaging modalities, the type and duration of previous therapies, and whether there are other known malignancies.

\section{E-PSMA}

The E-PSMA is a comprehensive guideline supported by the European Association of Nuclear Medicine and aims to develop a structured report for PSMA PET images and to harmonize diagnostic interpretation criteria (67). In the suggested structured EPSMA report, the visual description should relate PSMA uptake to background uptake in the blood pool, liver, and salivary glands on a visual scale of $0-3$. For image interpretation, the guideline panelists suggest a 5-point scale of confidence. The document also suggests the use of a standardized terminology in reporting PSMA PET findings and the adoption of a structured report.

\section{CONCLUSION}

PSMA ligands for PET imaging have been adopted at an unprecedented rate, resulting in a tremendous increase in published studies and trials. Most importantly, PSMA PET is now part of the diagnostic flowchart of PCa in international guidelines and has received the first regulatory approvals. Several PSMA radiotracers are now available and many more are under investigation, thus increasing the availability of PSMA PET imaging worldwide. Currently, the challenge lies in understanding the mechanisms behind PSMA expression and its influencing factors, either endogenous or exogenous. Furthermore, nuclear medicine physicians will have to familiarize themselves with a standardized reporting system, and a strict collaboration with the clinician remains vital for effective implementation of the PSMA PET imaging results. In short, from now on what we need is the production of reliable data on patient outcome at dedicated endpoints.

\section{DISCLOSURE}

No potential conflict of interest relevant to this article was reported.

\section{ACKNOWLEDGMENT}

We sincerely thank Dr. Alessandro Lambertini for the revision of the manuscript and his friendship.

\section{REFERENCES}

1. Siegel RL, Miller KD, Jemal A. Cancer statistics, 2019. CA Cancer J Clin. 2019;69:7-34.

2. Mapelli P, Incerti E, Ceci F, Castellucci P, Fanti S, Picchio M. ${ }^{11} \mathrm{C}$ - or ${ }^{18} \mathrm{~F}$-choline $\mathrm{PET} / \mathrm{CT}$ for imaging evaluation of biochemical recurrence of prostate cancer. J Nucl Med. 2016;57(suppl 3):43S-48S.

3. Ost P, Reynders D, Decaestecker K, et al. Surveillance or metastasis-directed therapy for oligometastatic prostate cancer recurrence: a prospective, randomized, multicenter phase II trial. J Clin Oncol. 2018;36:446-453.
4. Andriole GL, Kostakoglu L, Chau A, et al. The impact of positron emission tomography with ${ }^{18} \mathrm{~F}$-fluciclovine on the treatment of biochemical recurrence of prostate cancer: results from the LOCATE trial. J Urol. 2019;201:322-331.

5. Laudicella R, Albano D, Alongi P, et al. ${ }^{18} \mathrm{~F}$-Facbc in prostate cancer: a systematic review and meta-analysis. Cancers (Basel). 2019;11: 1348.

6. Touijer KA, Michaud L, Alvarez HAV, et al. Prospective study of the radiolabeled GRPR antagonist BAY86-7548 for positron emission tomography/computed tomography imaging of newly diagnosed prostate cancer. Eur Urol Oncol. 2019;2:166-173.

7. Perera M, Papa N, Roberts M, et al. Gallium-68 prostate-specific membrane antigen positron emission tomography in advanced prostate cancer: updated diagnostic utility, sensitivity, specificity, and distribution of prostate-specific membrane antigenavid lesions: a systematic review and meta-analysis. Eur Urol. 2020;77:403-417.

8. Paschalis A, Sheehan B, Riisnaes R, et al. Prostate-specific membrane antigen heterogeneity and DNA repair defects in prostate cancer. Eur Urol. 2019;76:469-478.

9. FDA approves first PSMA-targeted PET drug. J Nucl Med. 2021;62(2):11N.

10. Ghosh A, Heston WDW. Tumor target prostate specific membrane antigen (PSMA) and its regulation in prostate cancer. $J$ Cell Biochem. 2004;91:528-539.

11. Chen Y, Pullambhatla M, Foss CA, et al. 2-(3-\{1-carboxy-5- $\left[\left(6-\left[{ }^{18} \mathrm{~F}\right]\right.\right.$ fluoro-pyridine3-carbonyl)-amino]-pentyl $\}$-ureido)-pentanedioic acid, $\left[{ }^{18}\right.$ F $]$ DCFPyL, a PSMA-based PET imaging agent for prostate cancer. Clin Cancer Res. 2011;17:7645-7653.

12. Afshar-Oromieh A, Malcher A, Eder M, et al. PET imaging with a $\left[{ }^{68} \mathrm{Ga}\right]$ galliumlabelled PSMA ligand for the diagnosis of prostate cancer: biodistribution in humans and first evaluation of tumour lesions. Eur J Nucl Med Mol Imaging. 2013;40:486-495.

13. Afshar-Oromieh A, Hetzheim H, Kratochwil C, et al. The theranostic PSMA ligand PSMA-617 in the diagnosis of prostate cancer by PET/CT: biodistribution in humans, radiation dosimetry, and first evaluation of tumor lesions. J Nucl Med. $2015 ; 56: 1697-1705$.

14. Herrmann K, Bluemel C, Weineisen M, et al. Biodistribution and radiation dosimetry for a probe targeting prostate-specific membrane antigen for imaging and therapy. J Nucl Med. 2015;56:855-861.

15. Giesel FL, Hadaschik B, Cardinale J, et al. F-18 labelled PSMA-1007: biodistribution, radiation dosimetry and histopathological validation of tumor lesions in prostate cancer patients. Eur J Nucl Med Mol Imaging. 2017;44:678-688.

16. Wurzer A, Di Carlo D, Schmidt A, et al. Radiohybrid ligands: a novel tracer concept exemplified by ${ }^{18} \mathrm{~F}$ - or ${ }^{68} \mathrm{Ga}$-labeled rhPSMA inhibitors. J Nucl Med. 2020;61: 735-742.

17. Mottet N, van den Bergh RCN, Briers E, et al. EAU-EANM-ESTRO-ESUR-SIOG guidelines on prostate cancer: 2020 update. Part 1: screening, diagnosis, and local treatment with curative intent. Eur Urol. 2021;79:243-262.

18. Bodar YJL, Jansen BHE, van der Voorn JP, et al. Detection of prostate cancer with ${ }^{18} \mathrm{~F}$-DCFPyL PET/CT compared to final histopathology of radical prostatectomy specimens: is PSMA-targeted biopsy feasible? The DeTeCT trial. World J Urol. October 20, 2020 [Epub ahead of print].

19. Chen M, Zhang Q, Zhang C, et al. Combination of ${ }^{68}$ Ga-PSMA PET/CT and multiparametric MRI improves the detection of clinically significant prostate cancer: a lesion-by-lesion analysis. J Nucl Med. 2019;60:944-949.

20. Amin A, Blazevski A, Thompson J, et al. Protocol for the PRIMARY clinical trial, a prospective, multicentre, cross-sectional study of the additive diagnostic value of gallium-68 prostate-specific membrane antigen positron-emission tomography/ computed tomography to multiparametric magnetic reson: PRIMARY - a clinical trial protocol. BJU Int. 2020;125:515-524.

21. Bettermann AS, Zamboglou C, Kiefer S, et al. [ ${ }^{68}$ Ga-]PSMA-11 PET/CT and multiparametric MRI for gross tumor volume delineation in a slice by slice analysis with whole mount histopathology as a reference standard: implications for focal radiotherapy planning in primary prostate cancer. Radiother Oncol. 2019;141:214-219.

22. Scheltema MJ, Chang JI, Stricker PD, et al. Diagnostic accuracy of ${ }^{68} \mathrm{Ga}$-prostatespecific membrane antigen (PSMA) positron-emission tomography (PET) and multiparametric (mp)MRI to detect intermediate-grade intra-prostatic prostate cancer using whole-mount pathology: impact of the addition of ${ }^{68} \mathrm{Ga}-\mathrm{P}$. BJU Int. 2019;124:42-49.

23. Roberts MJ, Morton A, Donato P, et al. ${ }^{68} \mathrm{Ga}$-PSMA PET/CT tumour intensity preoperatively predicts adverse pathological outcomes and progression-free survival in localised prostate cancer. Eur J Nucl Med Mol Imaging. 2021;48:477-482.

24. Tu X, Zhang C, Liu Z, et al. The role of ${ }^{68} \mathrm{Ga}$-PSMA positron emission tomography/computerized tomography for preoperative lymph node staging in intermediate/high risk patients with prostate cancer: a diagnostic meta-analysis. Front Oncol. 2020;10:1365.

25. Hofman MS, Lawrentschuk N, Francis RJ, et al. Prostate-specific membrane antigen PET-CT in patients with high-risk prostate cancer before curative-intent surgery or radiotherapy (proPSMA): a prospective, randomised, multicentre study. Lancet. 2020;395:1208-1216.

26. Wondergem M, van der Zant FM, Broos WAM, et al. ${ }^{18}$ F-DCFPyL PET/CT for primary staging in 160 high-risk prostate cancer patients; metastasis detection rate, 
influence on clinical management and preliminary results of treatment efficacy. Eur J Nucl Med Mol Imaging. 2021;48:521-531.

27. Hope TA, Armstrong WR, Murthy V, et al. Accuracy of ${ }^{68}$ Ga-PSMA-11 for pelvic nodal metastasis detection prior to radical prostatectomy and pelvic lymph node dissection: a multicenter prospective phase III imaging study [abstract]. J Clin Oncol. 2020;38(suppl):5502.

28. de Feria Cardet RE, Hofman MS, Segard T, et al. Is prostate-specific membrane antigen positron emission tomography/computed tomography imaging cost-effective in prostate cancer: an analysis informed by the proPSMA trial. Eur Urol. 2021;79:413-418

29. Morigi JJ, Stricker PD, van Leeuwen PJ, et al. Prospective comparison of ${ }^{18}$ F-fluoromethylcholine versus ${ }^{68} \mathrm{Ga}$-PSMA PET/CT in prostate cancer patients who have rising PSA after curative treatment and are being considered for targeted therapy. J Nucl Med. 2015;56:1185-1190.

30. Calais J, Ceci F, Eiber M, et al. ${ }^{18}$ F-fluciclovine PET-CT and ${ }^{68}$ Ga-PSMA-11 PET-CT in patients with early biochemical recurrence after prostatectomy: a prospective, singlecentre, single-arm, comparative imaging trial. Lancet Oncol. 2019;20:1286-1294.

31. Han S, Woo S, Kim YJ, Suh CH. Impact of ${ }^{68} \mathrm{Ga}$-PSMA PET on the management of patients with prostate cancer: a systematic review and meta-analysis. Eur Urol. 2018;74:179-190.

32. Schmidt-Hegemann N-S, Fendler WP, Ilhan H, et al. Outcome after PSMA PET/ CT based radiotherapy in patients with biochemical persistence or recurrence after radical prostatectomy. Radiat Oncol. 2018;13:37

33. Farolfi A, Gafita A, Calais J, et al. ${ }^{68}$ Ga-PSMA-11 positron emission tomography detects residual prostate cancer after prostatectomy in a multicenter retrospective study. J Urol. 2019;202:1174-1181.

34. Meijer D, Donswijk ML, Bodar YJL, et al. Biochemical persistence of prostate-specific antigen after robot-assisted laparoscopic radical prostatectomy: tumor localizations using PSMA PET/CT imaging. J Nucl Med. November 6, 2020 [Epub ahead of print].

35. Cornford P, van den Bergh RCN, Briers E, et al. EAU-EANM-ESTRO-ESURSIOG guidelines on prostate cancer. Part II: 2020 update - treatment of relapsing and metastatic prostate cancer. Eur Urol. 2021;79:263-282.

36. Fendler WP, Calais J, Eiber M, et al. Assessment of ${ }^{68}$ Ga-PSMA-11 PET accuracy in localizing recurrent prostate cancer: a prospective single-arm clinical trial. JAMA Oncol. 2019;5:856-863.

37. Rauscher I, Düwel C, Haller B, et al. Efficacy, predictive factors, and prediction nomograms for ${ }^{68} \mathrm{Ga}$-labeled prostate-specific membrane antigen-ligand positronemission tomography/computed tomography in early biochemical recurrent prostate cancer after radical prostatectomy. Eur Urol. 2018;73:656-661.

38. Ceci F, Bianchi L, Borghesi M, et al. Prediction nomogram for ${ }^{68}$ Ga-PSMA-11 $\mathrm{PET} / \mathrm{CT}$ in different clinical settings of PSA failure after radical treatment for prostate cancer. Eur J Nucl Med Mol Imaging. 2020;47:136-146.

39. Emmett L, Tang R, Nandurkar R, et al. 3-year freedom from progression after ${ }^{68}$ Ga-PSMA PET/CT-triaged management in men with biochemical recurrence after radical prostatectomy: results of a prospective multicenter trial. $J$ Nucl Med. 2020;61:866-872.

40. Trabulsi EJ, Rumble RB, Jadvar H, et al. Optimum imaging strategies for advanced prostate cancer: ASCO guideline. J Clin Oncol . 2020;38:1963-1996.

41. Calais J, Czernin J, Cao M, et al. ${ }^{68}$ Ga-PSMA-11 PET/CT mapping of prostate cancer biochemical recurrence after radical prostatectomy in 270 patients with a PSA level of less than $1.0 \mathrm{ng} / \mathrm{mL}$ : impact on salvage radiotherapy planning. $J$ Nucl Med. 2018;59:230-237.

42. Calais J, Czernin J, Fendler WP, Elashoff D, Nickols NG. Randomized prospective phase III trial of ${ }^{68} \mathrm{Ga}$-PSMA-11 PET/CT molecular imaging for prostate cancer salvage radiotherapy planning. BMC Cancer [PSMA-SRT]. 2019;19:18.

43. Calais J, Armstrong WR, Kishan AU, et al. Update from PSMA-SRT trial NCT03582774: a randomized phase 3 imaging trial of prostate-specific membrane antigen positron emission tomography for salvage radiation therapy for prostate cancer recurrence powered for clinical outcome. Eur Urol Focus. December 29 2020 [Epub ahead of print].

44. Palma DA, Olson R, Harrow S, et al. Stereotactic ablative radiotherapy versus standard of care palliative treatment in patients with oligometastatic cancers (SABRCOMET): a randomised, phase 2, open-label trial. Lancet. 2019;393:2051-2058.

45. Parker CC, James ND, Brawley CD, et al. Radiotherapy to the primary tumour for newly diagnosed, metastatic prostate cancer (STAMPEDE): a randomised controlled phase 3 trial. Lancet. 2018;392:2353-2366.

46. Sundahl N, Gillessen S, Sweeney C, Ost P. When what you see is not always what you get: raising the bar of evidence for new diagnostic imaging modalities. Eur Urol. August 23, 2020 [Epub ahead of print].
47. Phillips R, Shi WY, Deek M, et al. Outcomes of observation vs stereotactic ablative radiation for oligometastatic prostate cancer: the ORIOLE phase 2 randomized clinical trial. JAMA Oncol. 2020;6:650-659.

48. Fendler WP, Weber M, Iravani A, et al. Prostate-specific membrane antigen ligand positron emission tomography in men with nonmetastatic castration-resistant prostate cancer. Clin Cancer Res. September 11, 2019 [Epub ahead of print].

49. Farolfi A, Hirmas N, Gafita A, et al. PSMA PET identifies PCWG3 target populations with superior accuracy and reproducibility when compared to conventional imaging: a multicenter retrospective study. J Nucl Med. September 11, 2020 [Epub ahead of print].

50. Ahmadzadehfar H, Azgomi K, Hauser S, et al. ${ }^{68}$ Ga-PSMA-11 PET as a gatekeeper for the treatment of metastatic prostate cancer with ${ }^{223} \mathrm{Ra}$ : proof of concept. $J$ Nucl Med. 2017;58:438-444.

51. Hofman MS, Violet J, Hicks RJ, et al. [ $\left.{ }^{177} \mathrm{Lu}\right]$-PSMA-617 radionuclide treatment in patients with metastatic castration-resistant prostate cancer (LuPSMA trial): a single-centre, single-arm, phase 2 study. Lancet Oncol. 2018;19:825-833.

52. Thang SP, Violet J, Sandhu S, et al. Poor outcomes for patients with metastatic castration-resistant prostate cancer with low prostate-specific membrane antigen (PSMA) expression deemed ineligible for ${ }^{177}$ Lu-labelled PSMA radioligand therapy. Eur Urol Oncol. 2019;2:670-676.

53. Seitz AK, Rauscher I, Haller B, et al. Preliminary results on response assessment using ${ }^{68} \mathrm{Ga}$-HBED-CC-PSMA PET/CT in patients with metastatic prostate cancer undergoing docetaxel chemotherapy. Eur J Nucl Med Mol Imaging. 2018;45:602-612.

54. Grubmüller B, Rasul S, Baltzer P, et al. Response assessment using $\left[{ }^{68} \mathrm{Ga}\right] \mathrm{Ga}-\mathrm{PSMA}$ ligand PET in patients undergoing systemic therapy for metastatic castration-resistant prostate cancer. Prostate. 2020;80:74-82.

55. Eisenhauer EA, Therasse P, Bogaerts J, et al. New response evaluation criteria in solid tumours: revised RECIST guideline (version 1.1). Eur J Cancer. 2009;45: 228-247.

56. Fanti S, Hadaschik B, Herrmann K, et al. Proposal for systemic-therapy response-assessment criteria at the time of PSMA PET/CT imaging: the PSMA PET progression criteria. J Nucl Med. 2020;61:678-682.

57. Scher HI, Morris MJ, Stadler WM, et al. Trial design and objectives for castration-resistant prostate cancer: updated recommendations from the Prostate Cancer Clinical Trials Working Group 3. J Clin Oncol . 2016;34:1402-1418.

58. Wright GL, Grob BM, Haley C, et al. Upregulation of prostate-specific membrane antigen after androgen-deprivation therapy. Urology. 1996;48:326-334.

59. Evans MJ, Smith-Jones PM, Wongvipat J, et al. Noninvasive measurement of androgen receptor signaling with a positron-emitting radiopharmaceutical that targets prostate-specific membrane antigen. Proc Natl Acad Sci USA. 2011;108:9578-9582.

60. Hope TA, Truillet C, Ehman EC, et al. ${ }^{68}$ Ga-PSMA-11 PET imaging of response to androgen receptor inhibition: first human experience. $J$ Nucl Med. 2017;58:81-84.

61. Emmett L, Yin C, Crumbaker M, et al. Rapid modulation of PSMA expression by androgen deprivation: serial ${ }^{68}$ Ga-PSMA-11 PET in men with hormone-sensitive and castrate-resistant prostate cancer commencing androgen blockade. J Nucl Med. 2019;60:950-954.

62. Ettala O, Malaspina S, Tuokkola T, et al. Prospective study on the effect of shortterm androgen deprivation therapy on PSMA uptake evaluated with ${ }^{68}$ Ga-PSMA$11 \mathrm{PET} / \mathrm{MRI}$ in men with treatment-naïve prostate cancer. Eur J Nucl Med Mol Imaging. 2020;47:665-673.

63. Afshar-Oromieh A, Debus N, Uhrig M, et al. Impact of long-term androgen deprivation therapy on PSMA ligand PET/CT in patients with castration-sensitive prostate cancer. Eur J Nucl Med Mol Imaging. 2018;45:2045-2054.

64. Vaz S, Hadaschik B, Gabriel M, Herrmann K, Eiber M, Costa D. Influence of androgen deprivation therapy on PSMA expression and PSMA-ligand PET imaging of prostate cancer patients. Eur J Nucl Med Mol Imaging. 2020;47:9-15.

65. Eiber M, Herrmann K, Calais J, et al. Prostate Cancer Molecular Imaging Standardized Evaluation (PROMISE): proposed miTNM classification for the interpretation of PSMA-ligand PET/CT. J Nucl Med. 2018;59:469-478.

66. Rowe SP, Pienta KJ, Pomper MG, Gorin MA. PSMA-RADS version 1.0: a step towards standardizing the interpretation and reporting of PSMA-targeted PET imaging studies. Eur Urol. 2018;73:485-487.

67. Ceci F, Oprea-Lager DE, Emmett L, et al. E-PSMA: the EANM standardized reporting guidelines v1.0 for PSMA PET. Eur J Nucl Med Mol Imaging. February 19, 2021 [Epub ahead of print]. 